\title{
Desirability Methods for Evaluating Visual Design
}

\author{
Julie Schiller and Edward De Guzman \\ Autodesk, Inc., One Market St., Suite 500, San Francisco, CA 94105 USA \\ \{julie.schiller, edward.deguzman\} @autodesk.com
}

\begin{abstract}
Previous studies show that traditional usability evaluation methods can be problematic for collecting feedback on visual design [1]. Desirability studies have been used by usability practitioners to collect feedback on the affective response to interactive systems, but none allow end users to contribute feedback in the language of visual design experts. We describe how we adapted two traditional user research techniques (card sort, directed storytelling) to collect feedback on visual design. We then compare and contrast the kinds of data gathered from these methods with data gathered in a think-aloud exercise. A mixed-methods research strategy that includes methods adapted for visual design offers a path to engaging end users in a conversation that results in concise and actionable feedback for visual designers.
\end{abstract}

\section{$1 \quad$ Introduction and Related Work}

Traditional usability evaluation methods can be difficult to adapt for accurately assessing visual design in later design iterations because feedback for visual design is less about the user's ability to accomplish tasks and more about the affective response to a design. Desirability research methods can collect attitudinal reactions to a design rather than behavioral reactions. The data collected helps designers understand why different design directions alter the emotion of a user experience.

While several groups have reported case studies of desirability studies $[1,2,4,5]$, it is unclear how desirability methods can be adapted to focus on visual design. Endusers of software are not usually expert visual designers and do not have the appropriate vocabulary to provide concise actionable feedback to visual designers as part of a user-centered design process. There is a need for projection techniques that give end users a framework to express deeper feedback on visual design and to start discussions with end users that typically couldn't happen using traditional usability research methods.

In [3] De Guzman and Schiller described the Visual Design Card Sort (VDCS) as a technique to assess visual design and presented several explorations of visualizations for presenting data from this method. The work in this paper builds upon this previous research in two ways. First, it proposes another method for assessing visual design -the Visual Design Mad Libs (VDML). Second, in order to better understand the benefits of VDCS and VDML as rapid user research methods, we compare the data collected from VDCS and VDML with the data collected from a think-aloud protocol study. Finally, we propose future directions for user research to partner and support visual design. 


\section{Visual Design Card Sort}

The Visual Design Reaction cards use a set of 75 Visual Design Reaction cards. 71 of the cards are a subset of the 118 Microsoft Product Reaction Cards from the Desirability Toolkit. Two researchers performed a card filtering exercise to inform and validate the set of cards, only keeping those that are relevant for providing feedback on the visual treatment of a design. Up to four additional cards can be used to add words that may be specific to the project or content being studied and/or to allow the participant to use their own word. Participants are then shown a set of images, one at a time. Once the image appears on the monitor, the participant has three to five minutes to select between three to five visual design reaction cards that best answers the question, "How does this look?" After the participant has selected a set of reaction cards, he/she is asked to explain why the card was chosen before moving on to the next image.

\section{$3 \quad$ Visual Design Mad Libs}

Visual Design Mad Libs is a hybrid of two research methods: directed storytelling and sentence completion tests, a semi-structured projective technique. To conduct a VDML, participants are shown a set of alternative visual designs along with control images. Once the image appears the participant has one minute to complete a one- to two-sentence statement where parts of the sentence(s) is left blank. For example, to assess the visual design of a product logo, the sentence to complete might be: "This is a logo for [company name]. The logo is a because

The design alternatives are placed in a randomized order among the control imagery such as popular competitor products. After the participant has completed the sentence, the researcher may ask follow-up questions to better understand the participant's response.

\section{Case Study: Evaluating Early Designs for an Autodesk Web Service}

During the development of three Autodesk web products, the Visual Design Card Sort and the Visual Design Mad Libs were used to collect feedback on a proposed visual redesign. The purpose of this research was to collect feedback from Autodesk users about a new look-and-feel and report on trends observed across the participants. The visual design team identified several design goals such as: "simple", "easy to use", "focused", "efficient", and "humanized" also identified a target emotional response -a "sense of wonder". 


\subsection{Materials}

Participants were shown a set of high-fidelity static images that reflected a redesign of the Autodesk brand. They were also shown a set of images for a visual redesign of a Web application for collaboration. The materials included an image of the applications landing page, an image of a list of documents stored in the Web service, and screenshots of how the application would be redesigned for a mobile device.

\subsection{Methodology}

Three different methods were used to collect feedback on these materials: the Visual Design Card Sort, Visual Design Mad Libs, and a think aloud protocol. These methods were used over three rounds of user research focused on visual design. Table 1 summarizes the methodologies used in each of the three rounds of research. The research strategy builds on [3] which focused solely on the VDCS and lacked a comparison of the data from VDCS with any other technique (row 1). In the second round of research (row 2) we compared VDCS with data from a think aloud protocol. Having data from both methods gave us a stronger picture of the reactions from the participants but left us unsure if it was the complete picture. In the third round of research (row 3) we used all three methods. We found the data collected from each method to be complementary: VDCS gave deep actionable specific feedback on design while VDML provided good direction on choosing between several alternative designs.

Table 1. Research methods used in each round of research

\begin{tabular}{|r|c|c|c|} 
& VDCS & Think Aloud & VDML \\
\hline Study 1 & $\boldsymbol{V}$ & & \\
\hline Study 2 & $\boldsymbol{V}$ & $\boldsymbol{V}$ & \\
\hline Study 3 & $\boldsymbol{V}$ & $\boldsymbol{V}$ & $\boldsymbol{V}$ \\
\hline
\end{tabular}

The Visual Design Mad Libs were used to collect feedback on three company logos. Participants were asked to describe what they saw and give a reason why the logo represents Autodesk in the following sentence:

"It is a because

Finally, the think aloud protocol was used as part of a semi-structured follow-up interview about the three variations of the redesigned Autodesk logo.

\subsection{Results}

Visual Design Card Sort: The VDCS is displayed as a word cloud reflecting the most popular cards chosen by participants in the study. Cards that were chosen more often appear in a larger font and bolder type. 
Visual Design Mad Libs:One participant's responses comparing three Autodesk logos:

- [Logo1] "It is a Folded 'A' because 'A' is for Autodesk. Kinda vague."

- [Logo 2] "It is a Bowtie or 3D glasses because you're making 3D TVs! Not good. Don't like the font"

- [Logo 3] "It is a perspective pyramid because you make software to build 3D designs"

Think aloud: Of the three logos presented to the participants, the strongest preference was for Logo 3:

- "More represents what Autodesk is known for. [It's] more recognizable as Autodesk."

- “...very precise, has gridlines."

- "Explains what we do. 3D design has volume."

\section{Discussion}

The past distance between visual design and HCI practices made it hard to get endusers engaged in the visual design development of an interactive product. Visual designers' complex vocabulary to discuss their work clashed with small sample user experience research feedback. However, in this case study, data was gathered both early and later in the development process. Accordingly, visual design was evaluated both holistically and in depth on specific pieces of the user interface.

HCI practitioners advocate including end-user feedback in the creation of user interfaces. Visual design for UIs has long the domain of experts due to the intricate, nuanced effect it has on user experience. In this paper, we advocate for more methods to express user experience along with brand or visual design needs. The techniques described contribute to deeper refinement, engagement, and a path to integrate these two fields.

\section{$6 \quad$ Conclusion and Future Work}

The use of desirability study methods has been documented in case studies but there has been little research and innovation in desirability research methods focused exclusively on assessing visual design. This is an opportunity for end-users to contribute to visual design goals. Techniques to make this feedback meaningful, actionable, and quick are needed.

Future directions include validation of some of the assumptions made which guided the design of this research method. We suspect that novice users are more subject to the acquiescence bias and would provide a significantly higher proportion of positive feedback than expert users. 
Another direction for future work is the compare the VDCS and VDML methods to expert review of visual designers, rather comparing it to the think-aloud method. Finally, these techniques offer projective surfaces for end-users. The VDCS and VDML methods are given to demonstrate that a shared vocabulary and evaluation framework can generate strong results for the visual design of UI development. Additionally, these findings engage in a discussion with visual design professionals on the opportunities for direct user feedback. Hopefully, these techniques will do justice to the intricate and nuanced work of visual design while providing a strong UCD focus.

\section{References}

1. Barnum, C.M., Palmer, L.A.: More than a feeling: understanding the desirability factor in user experience. Ext. Abstracts CHI 2010. ACM Press (2010)

2. Benedek, J., Miner, T.: Measuring Desirability: New Methods for Evaluating Desirability in a Usability Lab Setting. In: Proc. Usability Professionals Association Conference (2002)

3. De Guzman, E.S., Schiller, J.: How does this Look? Desirability Methods for Evaluating Visual Design. Ext. Abstracts of HCII 2011 (2011)

4. Petrie, H., Precious, J.: Measuring user experience of websites: think aloud protocols and an emotion word prompt list. Ext. Abstracts CHI 2010. ACM Press (2010)

5. Williams, D., Kelly, G., Anderson, L.: MSN 9: new user-centered desirability methods produce compelling visual design. In: Proc. CHI 2004. ACM Press (2004) 